\title{
VARIATION DE LAREPONSE AU STRESS SALIN CHEZ CINQ ESPECES DE LEGUMINEUSES
}

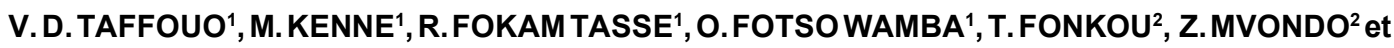 \\ A.AMOUGOU ${ }^{3}$
}

${ }^{1}$ Département de Biologie des Organismes Végétaux, Faculté des Sciences, Université de Douala, B.P. 24157 Douala,
Cameroun. E-mail : vtaffouo@yahoo.fr

2Département de Biologie Végétale, Faculté des Sciences, Université de Dschang, B.P. 96 Dschang, Cameroun.

${ }^{3}$ Département de Biologie et Physiologie Végétales; Faculté des Sciences, Université de Yaoundé 1, B.P. 812 Yaoundé, Cameroun.

\begin{abstract}
RESUME
Les effets de la salinité sur la croissance des organes de cinq espèces légumineuses ont été évalués afin d'identifier celles susceptibles d'être cultivées sur des terres plus ou moins salées. 4 niveaux de salinité $(0,50,100$ et $200 \mathrm{mM}$ de $\mathrm{NaCl})$ ont été testés. Ainsi, l'augmentation de la salinité entraîne chez Glycine max et Phaseolus vulgaris (glycophytes sensibles) la baisse de la biomasse sèche dès $50 \mathrm{mM}$ de $\mathrm{NaCl}$. Chez Mucuna poggei (halophyte facultative) et Vigna unguiculata (glycophyte moyennement tolérante) cette baisse s'observe dès $100 \mathrm{mM}$ de $\mathrm{NaCl}$. L'inhibition de la croissance des glycophytes sensibles est liée à une diminution de la consommation d'eau en milieu salé. Le blocage de $\mathrm{Na}^{+}$dans les racines représente le principal facteur responsable du déficit hydrique des feuilles. Chez Phaseolus adenanthus (halophyte), la production de matière sèche foliaire est peu affectée par la salinité. La résistance de cette espèce à $\mathrm{NaCl}$ s'explique par un bon cheminement du flux hydrique vers les feuilles, facilité par une augmentation des teneurs en $\mathrm{Na}^{+}$et $\mathrm{K}^{+}$dans le sens racines, tiges et feuilles. Par ailleurs, au niveau des feuilles la déficience en $\mathrm{K}^{+}$est liée à la salinité du milieu, qui limite la croissance chez les espèces étudiées. La tolérance de $V$. unguiculata, $M$. poggei, et $P$. adenanthus vis-à-vis du sel permet d'envisager leur utilisation sur des terres marginales plus ou moins salées.
\end{abstract}

Mots clés : stress salin, légumineuses, croissance, distribution ionique, Cameroun.

\author{
ABSTRACT \\ SALT STRESS RESPONSE VARIATION IN FIVE LEGUMINOUS PLANTS
}

In order to determine leguminous plants able to be used as pioneer plant species on more or less saline areas, effects of $\mathrm{NaCl}$ concentrations on the growth of organs of five leguminous wild or cultivated plants were evaluated. Plants were submitted to 4 levels of salt stress at the roots (0, 50, 100 and $200 \mathrm{mM}$ of $\mathrm{NaCl})$. The increase in salt concentration greatly reduces the production of dry mater at $50 \mathrm{mM}$ of $\mathrm{NaCl}$ in Glycine max and Phaseolus vulgaris (sensitive glycophytes). In Mucuna poggei (facultative halophyte) and Vigna unguiculata (moderately tolerant glycophyte) effect of salinity is observed for at least $100 \mathrm{mM}$ of $\mathrm{NaCl}$. Growth inhibition in the sensitive glycophytes is due to the decrease in water consumption within saline medium. The storage of $\mathrm{Na}^{+}$in roots can be responsible of the water deficit in leaves. In the natural halophyte Phaseolus adenanthus, the presence of salt does not affect the leaves' production of dry mater. This water flux is due to the significant storage of monovalent cation in leaves, facilitated by the increase in $\mathrm{Na}^{+}$and $\mathrm{K}^{+}$from roots to leaves. Moreover, the supply of $\mathrm{K}^{+}$in leaves is decreased because of the medium variation of the salt rate and therefore can be responsible of the limitation of the plant growth. The adapted behaviour of the glycophyte $(\mathrm{V}$. unguiculata) and of the two natural halophytes (M. poggei and P. adenanthus) suggest that they can be used as pioneer plant species able to grow on more or less saline medium.

Key words : salt stress, leguminous plants, plant growth, ionic distribution, Cameroon. 


\section{INTRODUCTION}

Dans le monde en général et au Cameroun en particulier, les zones semi-arides et les régions littorales présentent généralement de fortes teneurs en sel (Brun, 1981). Traditionnellement, les milieux naturels riches en chlorure de sodium créent pour les végétaux les conditions écologiques particulières au niveau des racines (forte pression osmotique et déséquilibre ionique). II en résulte pour le végétal des difficultés d'approvisionnement en eau et en éléments nutritifs indispensables ainsi que des risques d'empoisonnement par accumulation d'éléments tels que sodium ou chlore qui peuvent devenir toxiques à très forte dose (Tattini et al., 1995 ; Venkataraman et Venkataraman, 1995 ; Saintilan, 1998 ; Saadallaha et al., 2001).

Les différentes formes de réponses des plantes à l'effet défavorable du sel ont conduit les chercheurs (Rajesh et al., 1998 ; Sobrado, 1999a ; Sobrado et Ball, 1999) à distinguer deux groupes : (1) des espèces tolérantes dont les différentes phases du développement sont peu affectées ; (2) des espèces sensibles pour lesquelles la croissance est inhibée dès les plus faibles concentrations en sel. Cette distinction est particulièrement importante en agriculture où la possibilité de mise en valeur des terres salées passe par la sélection des plantes suivant leur degré de résistance à l'effet du sel.

La salinité est l'une des contraintes qui limite fortement le rendement des légumineuses à graines, particulièrement quand la croissance des plantes dépend de la fixation symbiotique de l'azote (Saadallaha et al., 2001a, 2001b ; Khadri et al., 2001). L'effet différentiel de la salinité sur la physiologie des divers organes végétaux reste peu connu. Dans le but d'intensifier et de diversifier la production des légumineuses alimentaires et/ou fourragères au Cameroun, nous recherchons des espèces qui s'adapteraient mieux aux milieux salés. Au Cameroun, les données concernant les espèces de plantes susceptibles de tolérer les variations de salinité du milieu, sont rares si l'on excepte les travaux de Brun $(1981,1987,1988)$ effectués sur la glycophyte (Phaseolus vulgaris) et I'halophyte naturelle (Canavalia obtusifolia) cultivée en condition semi-contrôlée de laboratoire. Par ailleurs les travaux de Tsoata et Taffouo (1997) ont traité essentiellement des possibilités d'absorption du sodium par les parties aériennes chez 4 espèces de légumineuses.
Entre les trois légumineuses traditionnellement cultivées au Cameroun , Glycine max, Phaseolus vulgaris et Vigna unguiculata) et les deux halophytes naturelles (Mucuna poggei et Phaseolus adenanthus), nous proposons de faire une étude comparative de leur compor-tement physiologique, afin de déterminer leur seuil de tolérance vis-à-vis des variations de la salinité du milieu.

\section{MATERIEL ET METHODES}

Les études ont été réalisées au Cameroun de Juin à Décembre 2000 à partir des graines de cinq légumineuses : (1) Glycine max est une plante herbacée annuelle fréquemment cultivée sur sol peu salé des régions du littoral et de l'Ouest du pays. La variété à graine jaune utilisée dans le présent travail a été récoltée dans la région du Littoral ; (2) Mucuna poggei est une plante grimpante pouvant atteindre une hauteur de 30 à $40 \mathrm{~m}$. Elle pousse spontanément sur sol sableux du littoral et la variété de couleur noire a été récoltée dans les environs de Douala; (3) Phaseolus adenanthus G.F.W. pousse spontanément sur les plages du littoral camerounais et supporte la sécheresse et la salinité du sol mieux que bien d'autres légumineuses (Duke, 1983). Les graines de cette espèce ont été récoltées sur les sables littoraux de la région de Kribi (Sud-Ouest Cameroun). (4) $P$. Vulgaris $L$. de variété «Pem Kou» à grains longs et rouge vineux est cultivé exclusivement à l'Ouest du Cameroun ; (5) Vigna unguiculata L. appelé «Niébé», cultivé dans les régions de l'Ouest, du Nord-Ouest et du SudEst du pays. La variété utilisée est celle issue de l'Ouest, à grain réniforme et de couleur marron (Pasquet, 1984).

\section{CULTURE ET MESURE DES PARAMETRES}

Les graines sont désinfectées par passage dans une solution d'hypochlorite de sodium à $10 \%$ pendant une heure, puis rincées abondamment et mises à germer entre deux feuilles de papier filtre imbibées d'eau déminéralisée. Les téguments des graines de $P$. adenanthus (extrêmement durs) sont entaillés à proximité du hile pour faciliter la germination. Après 3 jours, les graines germées sont réparties en 4 lots de 15 graines chacun et repiquées dans des bacs à réserve d'eau contenant du sable 
(nettoyé avec du $\mathrm{HCl}$ et rincé à l'eau déminéralisée). Chaque bac de culture est placé dans un local (température : $26,0 \pm 3,0{ }^{\circ} \mathrm{C}$; éclairement : 5000 lux pendant 12 heures ; humidité relative de l'air : 51 à $70 \%$ ). Les plantules qui se développent sont approvisionnées tous les 3 jours en solution nutritive composée de $0,4 \mathrm{mN}$ de $\mathrm{KNO}_{3}, 0,2 \mathrm{mN}$ de $\mathrm{KH}_{2} \mathrm{PO}_{4}, 1,0 \mathrm{mN}$ de $\mathrm{Ca}_{2} \mathrm{NO}_{3}$ et $0,4 \mathrm{mN}$ de $\mathrm{MgSO}_{4}$ (Wacquant, 1974).

Pour le lot de culture témoin $\left(S_{0}\right)$, la solution nutritive $(\mathrm{pH}=6)$ est dépourvue de $\mathrm{NaCl}$ tandis que pour les 3 lots expérimentaux $\left(S_{1}, S_{2}\right.$ et $\mathrm{S}_{3}$ ), elle est enrichie respectivement de 50, 100 et $200 \mathrm{mM}$ de $\mathrm{NaCl}$ (El-lklil et al., 2000). Au bout de 4 semaines, dans chaque milieu de culture deux séries de 5 plantes chacune sont prélevées au hasard dans la matinée. La première série permet de mesurer la biomasse sèche produite. Quant à la seconde série, les feuilles, les tiges et les racines sont isolées en vue d'évaluer les teneurs en eau, $\mathrm{Na}^{+}$et $\mathrm{K}^{+}$suivant la procédure décrite par Savouré (1980) et reprise par Tsoata et Taffouo (1997).

\section{ANALYSE STATISTIQUE}

Elles sont effectuées à l'aide du logiciel SigmaStat version 2.03. Les résultats sont donnés en terme de moyenne ( \pm déviation standard). La comparaison simultanée de plusieurs moyennes est réalisée suivant la procédure paramétrique d'ANOVA (test $F$ de Fisher) lorsque les conditions de normalité et d'égalité des variances sont vérifiées et les comparaisons des lots expérimentaux au lot témoin sont effectuées suivant la procédure de Dunnett. Dans le cas contraire le test multiple non paramétrique (test $\mathrm{H}$ de Kruskal-Wallis basé sur la médiane) est réalisé et les comparaisons au lot témoin sont effectués suivant la procédure de Dunn.

\section{RESULTATS}

\section{CROISSANCE DES PLANTULES}

En milieu témoin, la quantité de matière sèche produite est très élevée dans les feuilles et très faible dans les racines de Glycine max, Mucuna poggei et Phaseolus adenanthus. Par contre chez $P$. vulgaris et Vigna unguiculata, la quantité de matière sèche est très élevée dans les tiges (figure 1A).

Les données obtenues à partir des solutions nutritives enrichies en sel (Figure $1 \mathrm{~B}$ à 1D) permettent de constater que chez les glycophytes types $G$. max et $P$. vulgaris, la baisse de la quantité de matière sèche produite dans les différents organes végétaux est importante dès $50 \mathrm{mM}$ de $\mathrm{NaCl}$. Chez M. poggei cette baisse est significative dès $200 \mathrm{mM}$ de $\mathrm{NaCl}$ dans les racines et les tiges, tandis qu'au niveau des feuilles cette baisse s'observe dès $100 \mathrm{mM}$ de $\mathrm{NaCl}$. Chez $P$. adenanthus, la quantité de matière sèche foliaire n'est pas affectée par la variation du taux de salinité de la solution nutritive, contrairement aux racines et tiges où la baisse est significative dès $200 \mathrm{mM}$ de $\mathrm{NaCl}$. Chez V. unguiculata, la quantité de matière sèche produite est dans tous les cas, plus élevée dans les tiges que les autres organes. Les racines présentent dès $100 \mathrm{mM}$ de $\mathrm{NaCl}$ une baisse importante de la quantité de matière sèche tandis que l'effet du sel est moins marqué au niveau des tiges et des feuilles (tableau 1). 


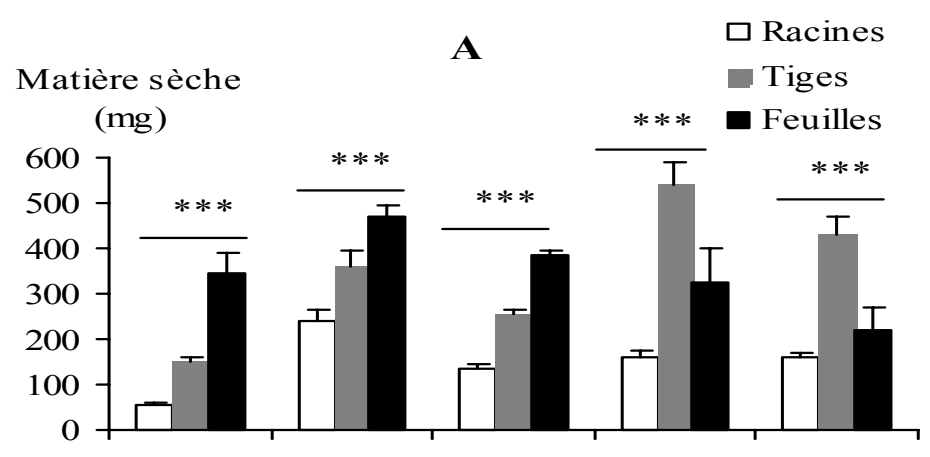

Matière sèche

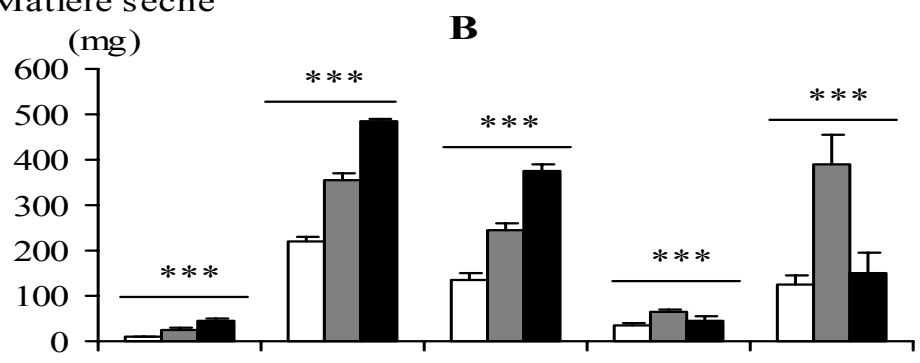

Matière sèche

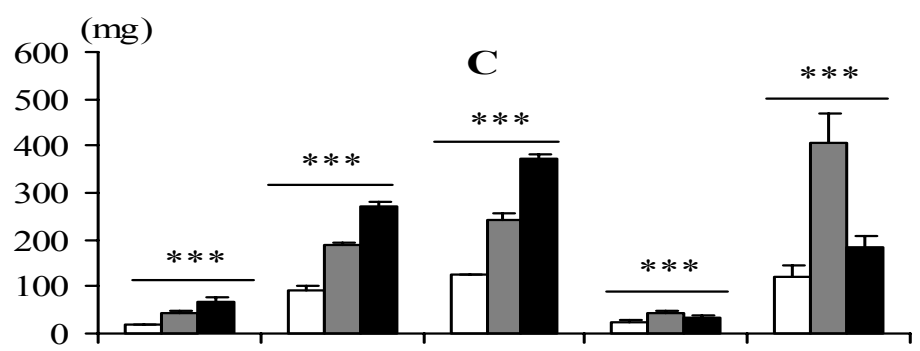

Matière sèche

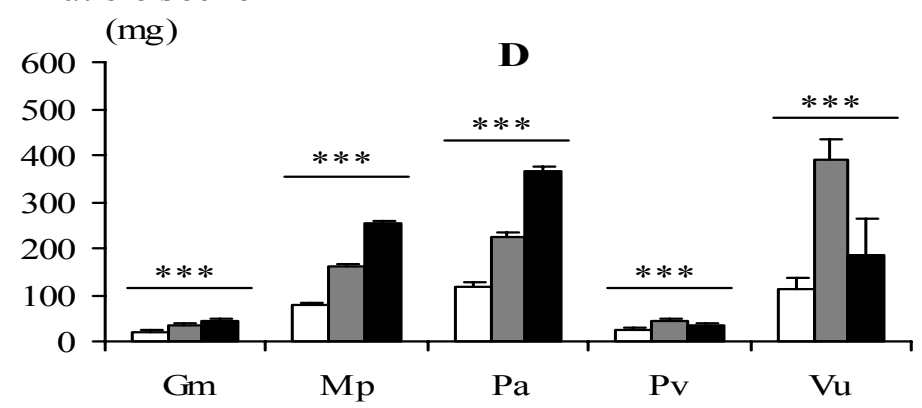

Figure 1 : Variation de la matière sèche produite dans les organes de cinq espèces de légumineuses.

$\mathrm{n}=5$ pour chaque espèce. $\mathrm{A}$ : milieu témoin $(0 \mathrm{mM}$ de $\mathrm{NaCl}) ; \mathrm{B}: 50 \mathrm{mM}$ de $\mathrm{NaCl} ; \mathrm{C}: 100 \mathrm{mM}$; $\mathrm{D}: 200 \mathrm{mM} ; \mathrm{Gm}=$ Glycine $\max , \mathrm{Mp}=$ Mucuna poggei, $\mathrm{Pa}=$ Phaseolus adenanthus, $\mathrm{Pv}=P$ vulgaris, $\mathrm{Vu}=$ Vigna unguiculata. ${ }^{* * *}=\mathrm{P}<0,001$.

Variation of the produced dry mater in organs of five leguminous plants.

$n=5$ for each species. $A$ : control medium (0 mM of $\mathrm{NaCl}) ; B: 50 \mathrm{mM}$ of $\mathrm{NaCl} ; \mathrm{C}: 100 \mathrm{mM} ; D$ : $200 \mathrm{mM} ; \mathrm{Gm}=$ Glycine max, $M p=$ Mucuna poggei ${ }_{2} P a=$ Phaseolus adenanthus, $P v=P$. vulgaris, Vu = Vigna unguiculata. ${ }^{* * *}=P<0,001$. 
Tableau 1 : Comparaisons des quantités de matière sèche chez 5 espèces de légumineuses $(n=5)$. Les probabilités significatives sont en gras.

$\mathrm{F}=$ test paramétrique d'ANOVA (les conditions de normalité et d'égalité des variances sont vérifiées) et les comparaisons au lot témoin sont faites suivant la procédure de Dunnett.

$\mathrm{H}=$ test non paramétrique de Kruskal-Wallis (les conditions de normalité et d'égalité des variances ne sont pas vérifiées) et les comparaisons au lot témoin sont effectués suivant la procédure de Dunn. Esp = espèces végétales, $\mathrm{Gm}=$ Glycine $\max , \mathrm{Mp}=$ Mucuna poggei, $\mathrm{Pa}=$ Phaseolus adenanthus, $\mathrm{Pv}=\mathrm{P}$. vulgaris, $\mathrm{Vu}=$ Vigna unguiculata.

Comparisons of the dry mater in organs of five leguminous plants $(n=5)$ using Fisher's one way ANOVA (when normality and equal variance passed) following by comparisons to the control group using Dunnett's method. Kruskal-Wallis one way ANOVA on ranks $(\mathrm{H}$ test) is performed when normality and equal variance tests failed and comparison procedures to the control group is performed using Dunn's method. Significant probabilities are in bold.

\begin{tabular}{|c|c|c|c|c|c|c|c|c|c|c|}
\hline \multirow{3}{*}{ Esp. } & \multicolumn{8}{|c|}{ Salinité du milieu de culture (en mM NaCl) } & \multirow{3}{*}{$\mathrm{F}_{(3,16)}$} & \multirow{3}{*}{$\mathrm{H}$} \\
\hline & \multicolumn{2}{|l|}{0} & \multicolumn{2}{|c|}{50} & \multicolumn{2}{|c|}{100} & \multicolumn{2}{|c|}{200} & & \\
\hline & $\mathrm{Moy} \pm \mathrm{DS}$ & Mé & $\operatorname{Moy} \pm \mathrm{DS}$ & Mé & $\operatorname{Moy} \pm \mathrm{DS}$ & Mé & $\operatorname{Moy} \pm \mathrm{DS}$ & Mé & & \\
\hline \multicolumn{11}{|c|}{ A- Racines } \\
\hline $\mathrm{Gm}$ & $\begin{array}{c}56,8 \\
\pm 4,8\end{array}$ & 58,0 & $\begin{array}{c}10,3 \\
\pm 1,6\end{array}$ & 9,5 & $\begin{array}{c}19,0 \\
\pm 2,0 *\end{array}$ & 20,0 & $\begin{array}{c}17,6 \\
\pm 4,8\end{array}$ & 20,0 & $\begin{array}{c}165,1 \\
* * *\end{array}$ & - \\
\hline $\mathrm{Mp}$ & $\begin{array}{l}241,4 \\
\pm 24,0\end{array}$ & 240,0 & $\begin{array}{l}221,8 \\
\pm 10,7\end{array}$ & 224,0 & $\begin{array}{l}93,8 \\
\pm 7,7\end{array}$ & 92,0 & $\begin{array}{l}80,2 \\
\pm 2,6\end{array}$ & $\begin{array}{c}81,0 \\
*\end{array}$ & - & $\begin{array}{l}16,5 \\
* * *\end{array}$ \\
\hline $\mathrm{Pa}$ & $\begin{array}{l}134,4 \\
\pm 10,8\end{array}$ & 132,0 & $\begin{array}{l}134,4 \\
\pm 16,9\end{array}$ & 128,0 & $\begin{array}{l}124,8 \\
\pm 3,3\end{array}$ & 124,0 & $\begin{array}{l}119,2 \\
\pm 5,9\end{array}$ & $120,0 *$ & - & $\begin{array}{c}8,92 \\
*\end{array}$ \\
\hline $\mathrm{Pv}$ & $\begin{array}{c}159,1 \\
\pm 13,5\end{array}$ & 165,2 & $\begin{array}{c}35,1 \\
\pm 4,1\end{array}$ & 33,4 & $\begin{array}{c}26,0 \\
\pm 3,6\end{array}$ & 24,0 & $\begin{array}{c}22,3 \\
\pm 6,5 \text { * }\end{array}$ & 19,4 & $\begin{array}{l}341,0 \\
* * *\end{array}$ & - \\
\hline $\mathrm{Vu}$ & $\begin{array}{c}158,7 \\
\pm 11,5\end{array}$ & 160,0 & $\begin{array}{c}126,5 \\
\pm 21,0\end{array}$ & 135,6 & $\begin{array}{c}123,2 \\
\pm 21,5\end{array}$ & 130,0 & $\begin{array}{c}110,1 \\
\pm 27,8\end{array}$ & 104,7 & $\begin{array}{c}4,7 \\
*\end{array}$ & - \\
\hline \multicolumn{11}{|c|}{ B- Tiges } \\
\hline $\mathrm{Gm}$ & $\begin{array}{l}147,6 \\
\pm 10,8\end{array}$ & 147,0 & $\begin{array}{c}26,2 \\
\pm 1,9\end{array}$ & 27,0 & $\begin{array}{c}42,4 \\
\pm 5,3\end{array}$ & 41,5 & $\begin{array}{c}34,1 \\
\pm 3,1\end{array}$ & 32,5 & $\begin{array}{c}412,4 \\
* * *\end{array}$ & - \\
\hline $\mathrm{Mp}$ & $\begin{array}{l}359,0 \\
\pm 34,0\end{array}$ & 240,0 & $\begin{array}{l}354,0 \\
\pm 13,7\end{array}$ & 224,0 & $\begin{array}{l}187,0 \\
\pm 5,9\end{array}$ & 92,0 & $\begin{array}{l}160,0 \\
\pm 3,5\end{array}$ & $\begin{array}{c}81,0 \\
*\end{array}$ & - & $\begin{array}{l}16,1 \\
* * *\end{array}$ \\
\hline $\mathrm{Pa}$ & $\begin{array}{l}253,6 \\
\pm 10,2\end{array}$ & 252,0 & $\begin{array}{l}243,6 \\
\pm 18,1\end{array}$ & 242,0 & $\begin{array}{l}243,2 \\
\pm 11,9\end{array}$ & 240,0 & $\begin{array}{l}224,4 \\
\pm 8,8 *\end{array}$ & 224,0 & $\begin{array}{c}4,5 \\
*\end{array}$ & - \\
\hline $\mathrm{Pv}$ & $\begin{array}{l}538,8 \\
\pm 50,4\end{array}$ & 560,4 & $\begin{array}{l}62,5 \\
\pm 9,4\end{array}$ & 61,3 & $\begin{array}{l}45,3 \\
\pm 5,0\end{array}$ & $\begin{array}{l}47,0 \\
*\end{array}$ & $\begin{array}{l}43,7 \\
\pm 7,1\end{array}$ & $\begin{array}{c}40,9 \\
*\end{array}$ & - & $\begin{array}{r}14,9 \\
* *\end{array}$ \\
\hline $\mathrm{Vu}$ & $\begin{array}{l}429,6 \\
\pm 41,5\end{array}$ & 447,5 & $\begin{array}{l}392,1 \\
\pm 63,3\end{array}$ & 373,9 & $\begin{array}{l}405,5 \\
\pm 63,3\end{array}$ & 419,7 & $\begin{array}{l}392,9 \\
\pm 44,0\end{array}$ & 383,0 & $\begin{array}{c}0,55 \\
\text { ns }\end{array}$ & - \\
\hline \multicolumn{11}{|c|}{ C- Feuilles } \\
\hline $\mathrm{Gm}$ & $\begin{array}{l}346,0 \\
\pm 42,6\end{array}$ & 324,0 & $\begin{array}{c}46,8 \\
\pm 4,7\end{array}$ & 45,0 & $\begin{array}{c}69,8 \\
\pm 9,3\end{array}$ & 70,0 & $\begin{array}{c}43,0 \\
\pm 4,3\end{array}$ & 43,0 & $\begin{array}{l}222,5 \\
* * *\end{array}$ & - \\
\hline $\mathrm{Mp}$ & $\begin{array}{l}471,0 \\
\pm 22,0\end{array}$ & 475,0 & $\begin{array}{c}484,8 \\
\pm 7,0\end{array}$ & 485,0 & $\begin{array}{c}271,8 \\
\pm 10,6\end{array}$ & 274,0 & $\begin{array}{c}256,0 \\
\pm 4,3\end{array}$ & 254,0 & $\begin{array}{l}460,7 \\
* * *\end{array}$ & - \\
\hline $\mathrm{Pa}$ & $\begin{array}{l}383,2 \\
\pm 12,5\end{array}$ & 384,0 & $\begin{array}{l}376,0 \\
\pm 11,7\end{array}$ & 376,0 & $\begin{array}{l}372,0 \\
\pm 8,9\end{array}$ & 372,0 & $\begin{array}{c}365,6 \\
\pm 9,6\end{array}$ & 368,0 & $\begin{array}{c}2,3 \\
\text { ns }\end{array}$ & - \\
\hline $\mathrm{Pv}$ & $\begin{array}{l}326,6 \\
\pm 73,0\end{array}$ & 312,0 & $\begin{array}{l}46,7 \\
\pm 7,8\end{array}$ & 45,0 & $\begin{array}{l}34,5 \\
\pm 6,3\end{array}$ & $\underset{*}{33,4}$ & $\begin{array}{l}33,6 \\
\pm 3,9\end{array}$ & $\begin{array}{c}33,6 \\
*\end{array}$ & - & $\begin{array}{l}14,9 \\
* *\end{array}$ \\
\hline $\mathrm{Vu}$ & $\begin{array}{l}220,3 \\
\pm 48,4\end{array}$ & 210,0 & $\begin{array}{l}148,5 \\
\pm 48,1\end{array}$ & 162,8 & $\begin{array}{l}184,3 \\
\pm 25,2\end{array}$ & 190,0 & $\begin{array}{l}183,4 \\
\pm 81,2\end{array}$ & 170,0 & $\begin{array}{l}1,4 \\
\mathrm{~ns}\end{array}$ & - \\
\hline
\end{tabular}

ns $=\mathrm{P}>0,05 ;{ }^{*}=\mathrm{P}<0,05 ;{ }^{* *}=\mathrm{P}<0,01 ;{ }^{* *}=\mathrm{P}<0,001$.

\section{DISTRIBUTION DE NA ${ }^{+} \mathrm{ET} \mathrm{K}^{+}$}

Les teneurs en $\mathrm{Na}^{+}$des organes végétaux des espèces étudiées montrent qu'en milieu témoin, elles sont inférieures à $50 \mu$ éq. $\mathrm{g}^{-1} \mathrm{MS}$ (figure. $2 \mathrm{~A}$ ). L'apport de $\mathrm{NaCl}$ dans le milieu de culture entraîne une augmentation de ces teneurs dans tous les organes des espèces étudiées. Contrairement à $P$. adenanthus, ces teneurs sont élevées au niveau des racines et faibles dans les feuilles de G. max, M. poggei, $P$. vulgaris et $V$. unguiculata (figure $2 \mathrm{~B}, 2 \mathrm{C}$ et $2 \mathrm{D}$ ). 
Quant aux teneurs en $\mathrm{K}^{+}$, elles sont élevées dans les feuilles et faibles dans les racines de toutes les espèces étudiées (figure 3A). L'apport de $\mathrm{NaCl}$ dans le milieu de culture entraîne une baisse importante de ces teneurs dans tous les organes considérés (figure 3B, $3 C$ et $3 D$ ). Toutefois pour une salinité de 100 $\mathrm{mM}$ (figure 3C) ces teneurs diminuent considérablement dans les racines et les tiges de $P$. adenanthus.
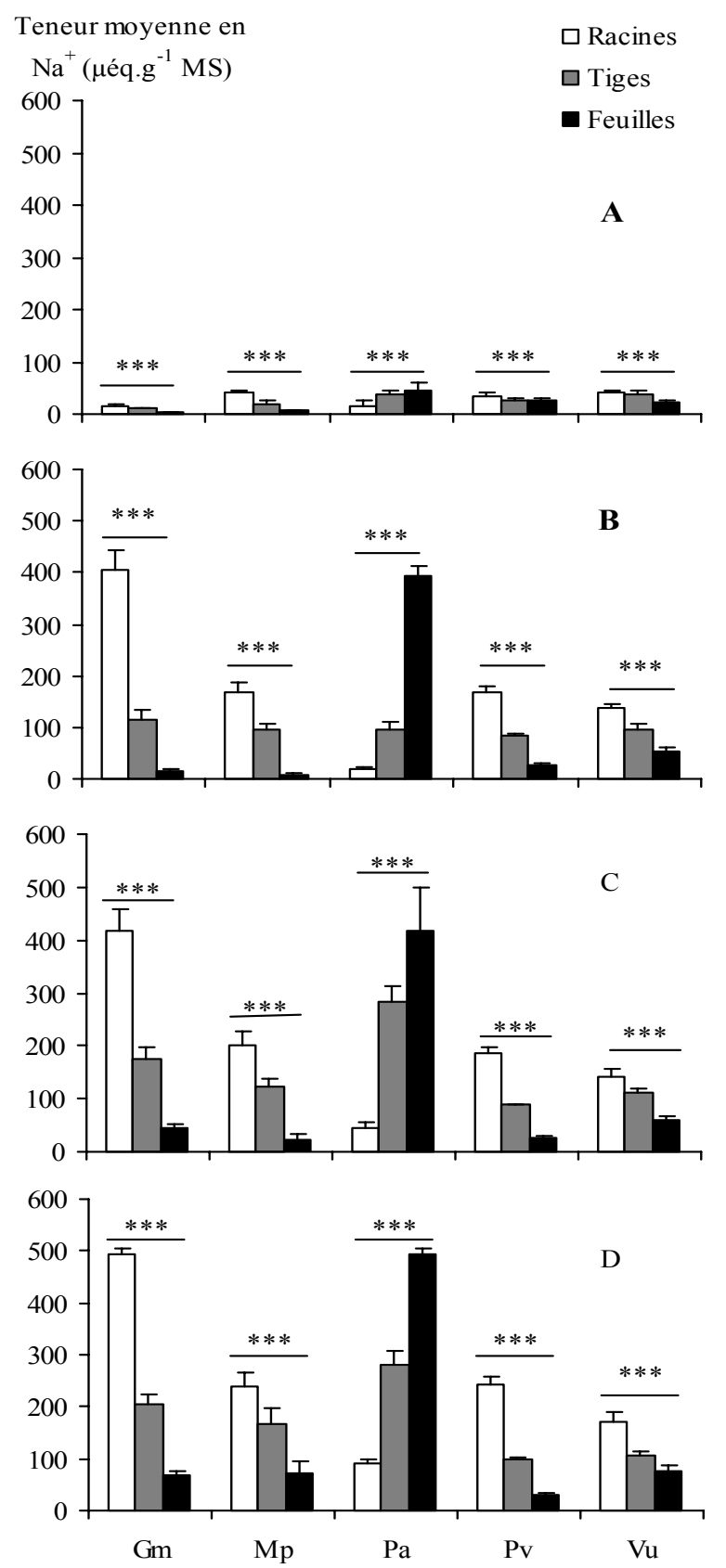

Figure 2 : Distribution de $\mathrm{Na}+$ dans les organes de cinq espèces de légumineuses.

$\mathrm{n}=5$ pour chaque espèce. $\mathrm{A}$ : milieu témoin $(0 \mathrm{mM}$ de $\mathrm{NaCl}) ; \mathrm{B}: 50 \mathrm{mM}$ de $\mathrm{NaCl} ; \mathrm{C}: 100 \mathrm{mM}$; $\mathrm{D}: 200 \mathrm{mM} ; \mathrm{Gm}=$ Glycine max, $\mathrm{Mp}=$ Mucuna poggei, $\mathrm{Pa}=$ Phaseolus adenanthus, $\mathrm{Pv}=P$. vulgaris, $\mathrm{Vu}=$ Vigna unguiculata. ${ }^{* \star *}=\mathrm{P}<0,001$.

Salt effets on distribution of $\mathrm{Na}+$ in organs of five leguminous plants.

$n=5$ for each species. $A$ : control medium (0 mM of $\mathrm{NaCl}) ; B: 50 \mathrm{mM}$ of $\mathrm{NaCl} ; C: 100 \mathrm{mM} ; D$ : $200 \mathrm{mM} ; \mathrm{Gm}=$ Glycine $\max , \mathrm{Mp}=$ Mucuna poggei, $\mathrm{Pa}=$ Phaseolus adenanthus, $P v=P$. vulgaris, $V u=$ Vigna unguiculata. ${ }^{* * *}=P<0,001$. 

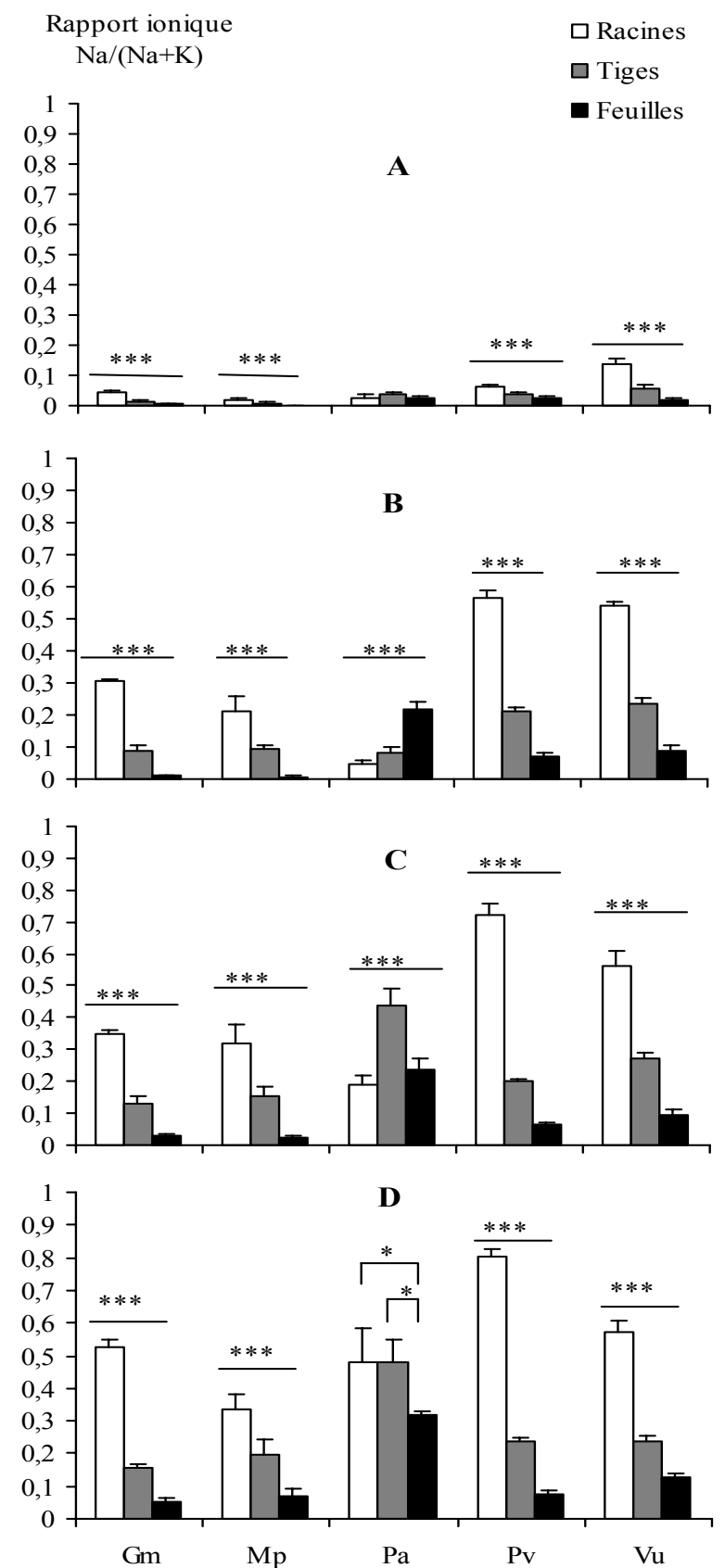

Figure 3 : Distribution de $\mathrm{K}+$ dans les organes de cinq espèces de légumineuses.

$\mathrm{n}=5$ pour chaque espèce végétale. $\mathrm{A}$ : milieu témoin $(0 \mathrm{mM}$ de $\mathrm{NaCl}) ; \mathrm{B}: 50 \mathrm{mM}$ de $\mathrm{NaCl} ; \mathrm{C}$ : $100 \mathrm{mM}$; D : $200 \mathrm{mM} ; \mathrm{Gm}=$ Glycine $\max , \mathrm{Mp}=$ Mucuna poggei, $\mathrm{Pa}=$ Phaseolus adenanthus, $\mathrm{Pv}$ $=P$. vulgaris, $\mathrm{Vu}=$ Vigna unguiculata. ${ }^{* *}=\mathrm{P}<0,001$.

Salt effets on distribution of $K+$ in organs of five leguminous plants.

$\mathrm{n}=5$ for each species. A : control medium ( $0 \mathrm{mM}$ of $\mathrm{NaCl})$; $\mathrm{B}: 50 \mathrm{mM}$ of $\mathrm{NaCl}$; $\mathrm{C}: 100 \mathrm{mM}$; $\mathrm{D}$ : $200 \mathrm{mM} ; \mathrm{Gm}=$ Glycine max, $\mathrm{Mp}=$ Mucuna poggei, $\mathrm{Pa}=$ Phaseolus adenanthus, $\mathrm{Pv}=P$. vulgaris, $\mathrm{Vu}=$ Vigna unguiculata. ${ }^{* * *}=\mathrm{P}<0,001$.

\section{Accumulation ionique globale}

En milieu témoin, les rapports ioniques obtenus suggèrent de faibles teneurs en $\mathrm{Na}^{+}$au niveau des organes végétaux des espèces étudiées. Toutefois ce rapport est voisin de 0,1 dans les racines de $V$. unguiculata (figure 4A). L'apport de $\mathrm{NaCl}$ dans le milieu de culture entraîne une augmentation du rapport ionique dans les différents organes des espèces étudiées. Par ailleurs chez G. max, M. poggei, P. vulgaris et $V$. unguiculata, ces rapports sont élevés dans 
les racines et faibles dans les feuilles, contrairement à $P$. adenanthus où ils sont plutôt élevés au niveau des feuilles, des tiges ou des tiges et des racines respectivement pour 50, 100 et $200 \mathrm{mM}$ de $\mathrm{NaCl}$ (figure 4B, 4C et 4D).

\section{ETAT HYDRIQUE}

Par rapport au milieu témoin, l'apport de $\mathrm{NaCl}$ dans le milieu de culture entraîne une baisse des teneurs en eau dans tous les organes des espèces étudiées. Toutefois, quel que soit le taux de $\mathrm{NaCl}$ cette baisse est non significative chez $P$. adenanthus et significative dès 50 et $100 \mathrm{mM}$ de $\mathrm{NaCl}$ respectivement chez les espèces sensibles (G. max et $P$. vulgaris) et chez les espèces moyennement tolérantes (M. poggei et $V$. unguiculata) (tableau 2).
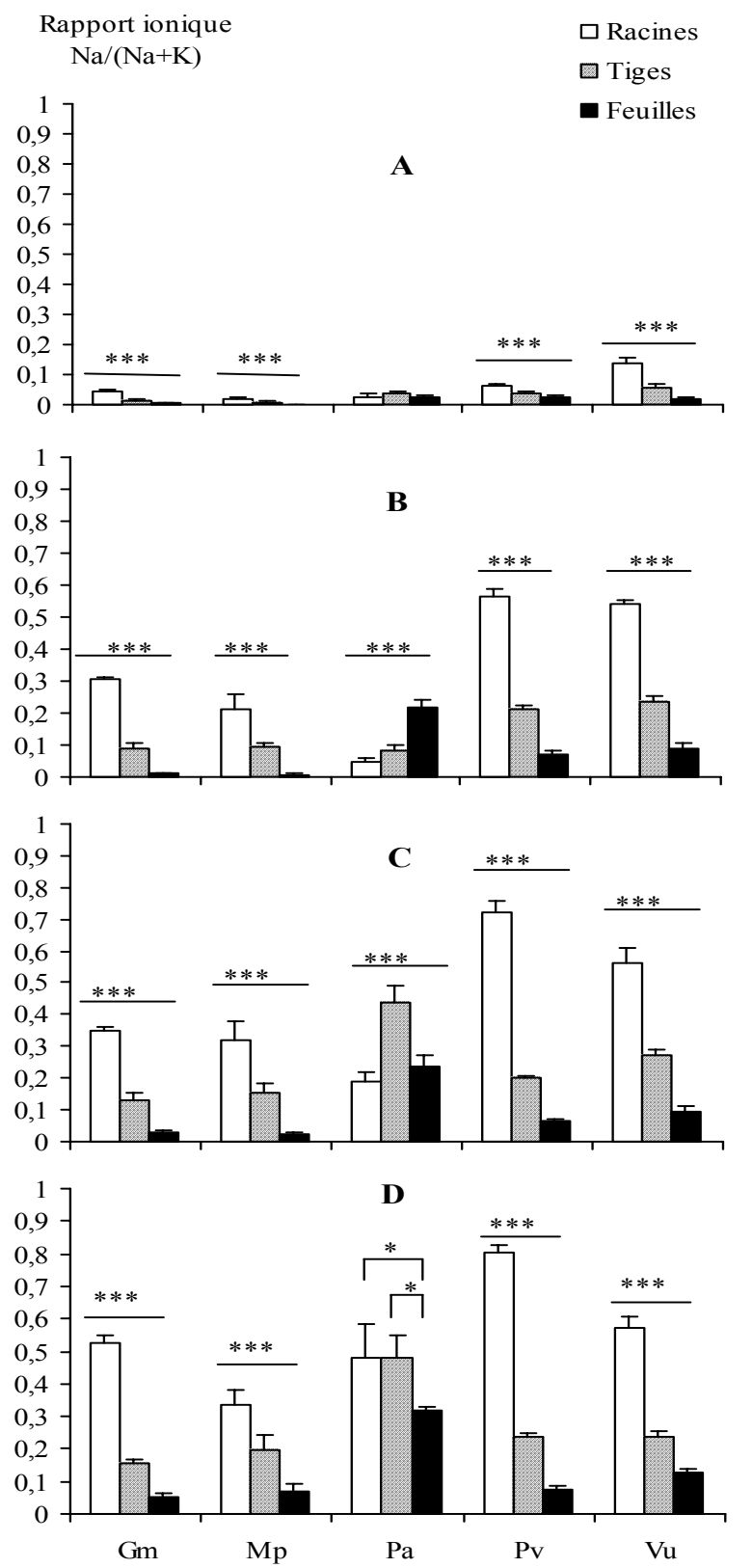

Figure 4 : Effets de $\mathrm{NaCl}$ sur le rapport $\mathrm{Na} /(\mathrm{Na}+\mathrm{K})$ des organes végétaux chez cinq espèces de légumineuses.

$\mathrm{n}=5$ pour chaque espèce végétale. $\mathrm{A}$ : milieu témoin $(0 \mathrm{mM}$ de $\mathrm{NaCl}) ; \mathrm{B}: 50 \mathrm{mM}$ de $\mathrm{NaCl} ; \mathrm{C}: 100 \mathrm{mM} ; \mathrm{D}: 200$ $\mathrm{mM} ; \mathrm{Gm}=$ Glycine max, $\mathrm{Mp}=$ Mucuna poggei, $\mathrm{Pa}=$ Phaseolus adenanthus, $\mathrm{Pv}=P$. vulgaris, $\mathrm{Vu}=$ Vigna unguiculata. ${ }^{* \star *}=\mathrm{P}<0,001$

Salt effets on the ionic ratio $\mathrm{Na} /(\mathrm{Na}+\mathrm{K})$ of vegetal organs of five leguminous plants

$n=5$ for each species. $A$ : control medium $(0 \mathrm{mM}$ of $\mathrm{NaCl}) ; B: 50 \mathrm{mM}$ of $\mathrm{NaCl}: C: 100 \mathrm{mM} ; D: 200 \mathrm{~m}: \mathrm{Gm}$ $=$ Glycine $\max , M p=$ Mucuna poggei, $P a=$ Phaseolus adenanthus, $P v=P$. vulgaris, $V u=$ Vigna unguiculata. ${ }^{* *}=P<0,001$. 
Tableau 2 : Effet de $\mathrm{NaCl}$ sur la teneur en eau des organes de 5 espèces de légumineuses $(n=5)$.

Les résultats sont analysés à l'aide du test paramétrique d'ANOVA (les conditions de normalité et d'égalité des variances sont vérifiées). Les probabilités significatives sont en gras. Pour les comparaisons analytiques, les lots expérimentaux sont comparés au lot témoin suivant la procédure de Dunnett.

Salt effects on the water content of organs of five leguminous plants $(n=5)$ using Fisher' one way ANOVA (normality and equal variance passed). Pairwise comparisons to the control group are performed using Dunnett's method. Significant probabilities are in bold.

\begin{tabular}{|c|c|c|c|c|c|}
\hline \multirow{2}{*}{ Espèces } & \multicolumn{4}{|c|}{ Salinité du milieu de culture (en $\mathrm{mM}$ de $\mathrm{NaCl}$ ) } & \multirow{2}{*}{$\frac{\text { ANOVA }}{\mathrm{F}_{(3,16)}}$} \\
\hline & 0 & 50 & 100 & 200 & \\
\hline \multicolumn{6}{|l|}{ 1. Racines } \\
\hline Glycine $\max$ & $86,3 \pm 1,0$ & $79,0 \pm 2,6 *$ & $74,4 \pm 3,0 *$ & $65,0 \pm 3,8 *$ & $50,12 * * *$ \\
\hline Mucuna poggei & $79,0 \pm 2,0$ & $77,4 \pm 1,2$ & $73,2 \pm 2,4 *$ & $71,8 \pm 2,9 *$ & $11,91 * * *$ \\
\hline Phaseolus adenanthus & $74,6 \pm 1,7$ & $72,9 \pm 2,0$ & $73,6 \pm 0,7$ & $72,6 \pm 1,9$ & $1,41 \mathrm{~ns}$ \\
\hline P. vulgaris & $89,1 \pm 4,3$ & $80,3 \pm 0,6 *$ & $70,0 \pm 3,1 *$ & $66,3 \pm 2,8 *$ & $58,29 * * *$ \\
\hline $\begin{array}{l}\text { Vigna unguiculata } \\
\text { 2. Tiges }\end{array}$ & $90,6 \pm 0,5$ & $87,7 \pm 2,6$ & $84,2 \pm 1,6 *$ & $78,3 \pm 2,9 *$ & $30,63 * * *$ \\
\hline G. $\max$ & $86,3 \pm 1,0$ & $\mathbf{7 4 , 4} \pm \mathbf{4 , 0} *$ & $71,8 \pm 2,9 *$ & $61,0 \pm 3,2 *$ & $60,61 * * *$ \\
\hline M. poggei & $83,4 \pm 1,6$ & $82,0 \pm 0,8$ & $81,6 \pm 1,0 *$ & $79,2 \pm 0,6 *$ & $13,09 * * *$ \\
\hline P. adenanthus & $80,7 \pm 0,8$ & $79,2 \pm 1,3$ & $79,0 \pm 1,6$ & $78,8 \pm 1,4$ & $2,21 \mathrm{~ns}$ \\
\hline P. vulgaris & $92,3 \pm 0,3$ & $74,2 \pm 2,7 *$ & $68,5 \pm 2,7 *$ & $63,1 \pm 2,3 *$ & $161,18 * * *$ \\
\hline $\begin{array}{l}V \text {. unguiculata } \\
\text { 3. Feuilles }\end{array}$ & $92,2 \pm 0,4$ & $92,1 \pm 0,3$ & $89,2 \pm 1,6 *$ & $87,8 \pm 2,7 *$ & $9,60 * * *$ \\
\hline G. $\max$ & $86,0 \pm 2,9$ & $65,2 \pm 3,9 *$ & $52,0 \pm 3,7 *$ & $42,5 \pm 5,0 *$ & $114,91 * * *$ \\
\hline M. poggei & $83,1 \pm 0,6$ & $82,2 \pm 0,2$ & $77,5 \pm 1,2 *$ & $76,0 \pm 1,8 *$ & $51,46 * * *$ \\
\hline P. adenanthus & $76,0 \pm 1,6$ & $76,3 \pm 0,8$ & $75,3 \pm 0,6$ & $75,0 \pm 2,2$ & $0,81 \mathrm{~ns}$ \\
\hline P. vulgaris & $91,5 \pm 0,9$ & $67,9 \pm 2,0 *$ & $65,3 \pm 2,7 *$ & $46,9 \pm 2,2 *$ & $384,05 * * *$ \\
\hline V. unguiculata & $84,7 \pm 0,6$ & $83,2 \pm 0,4$ & $80,7 \pm 0,5 *$ & $80,1 \pm 3,3 *$ & $8,13 * *$ \\
\hline
\end{tabular}

$\mathrm{ns}=\mathrm{P}>0,05 ;{ }^{*}=\mathrm{P}<0,05 ;{ }^{* *}=\mathrm{P}<0,01 ;{ }^{* *}=\mathrm{P}<0,001$.

\section{DISCUSSION}

\section{CROISSANCE DES PLANTULES}

L'apport de $\mathrm{NaCl}$ dans le milieu de culture entraîne la baisse de la quantité de matière sèche des différents organes végétaux chez Glycine max et Phaseolus vulgaris. Cette baisse serait liée à une inhibition de l'activité de certaines enzymes dans les organes impliqués. L'effet négatif du stress salin sur la croissance des végétaux est largement décrit chez plusieurs espèces cultivées (Jacoby, 1964 ; Greenway et Munns, 1980 ; Brun 1987). Certains auteurs lient la réponse au stress salin à la variation génotypique des plantes (Saadallaha et al., 2001a et 2001b) tandis que d'autres signalent une inhibition de l'activité de certaines enzymes entre autre la nitrogénase et la glutamine synthétase (Khadri et al., 2001). G. max et P. vulgaris, peuvent ainsi être qualifiées de "glycophytes sensibles» dont le seuil de tolérance se situe entre 0 et $50 \mathrm{mM}$ de $\mathrm{NaCl}$ (Levitt, 1980). Par ailleurs, chez ces deux espèces végétales l'effet défavorable du sel sur la production de la matière sèche est plus marqué au niveau des parties aériennes que les racines.

Chez $P$. adenanthus, $M$. poggei et $V$. unguiculata, l'effet défavorable du sel est important au niveau de l'appareil racinaire. Ceci rappelle les résultats observés chez la "glycophyte moyennement tolérante» Neirium oleander, et chez deux halophytes littorales Lavatera arborea et Canavalia obtusifolia (Hajji, 1979 ; Okusanya, 1980 ; Brun, 1988). Signalons que les deux espèces littorales ( $P$. adenanthus et $M$. poggei) présentent de larges feuilles et développent un système nodulaire abondant tandis que $V$. unguiculata présente des feuilles épaisses comparables à celles de I'halophyte naturelle C. obtusifolia (Brun, 1988). Ces caractéristiques phénotypiques sont reconnues comme étant responsables de la relative tolérance des végétaux au sel (Saadallaha et al., 2001a). 


\section{DISTRIBUTION DE $\mathrm{Na}^{+}$et $\mathbf{K}^{+}$}

Chez les espèces végétales étudiées, les teneurs en $\mathrm{Na}^{+}$des organes augmentent avec la concentration en sel du milieu. Toutefois, ces teneurs sont élevées dans les racines et faibles dans les feuilles de G. max, M. poggei, P. vulgaris et $V$. unguiculata. Ceci suggère que le sodium absorbé par les racines est peu transféré vers les parties aériennes des plantules. Le blocage des ions sodium au niveau des racines permet à la plante d'éviter l'envahissement des feuilles par ces éléments toxiques susceptibles d'empêcher l'activité photosynthétique, suite à la réduction de l'ouverture des stomates et des échanges gazeux (Berger, 1978 ; Tattini et al., 1995 ; Rajesh et al., 1998 ; Sobrado, 1999a, 1999b). Ces résultats se rapprochent de ceux décrits chez plusieurs glycophytes et halophytes (Shapman, 1976 ; Lessani et Marschner, 1978 ; Slama et Bouazi, 1978; Brun, 1987). Nos observations sont pourtant contraires aux résultats de Slama (1987) qui signale plutôt une exclusion passive des ions sodium au niveau des feuilles de plusieurs glycophytes. La particularité de nos observations est que le système racinaire des plantules empêche la migration accentuée des ions sodium vers les parties sommitales du végétal (feuilles). Par ailleurs plusieurs travaux montrent qu'au niveau des tiges des plantules de $P$. vulgaris et Prosopis farcata, l'hypocotyle et le système conducteur interviennent très souvent dans le contrôle de la migration de $\mathrm{Na}^{+}$vers les feuilles (Jacoby, 1964 ; Scheidecker, 1978, Levitt, 1980). Le comportement de $M$. poggei et $V$. unguiculata dont la croissance n'est ralentie qu'à partir de $100 \mathrm{mM}$ de $\mathrm{NaCl}$ est comparable à celui de Nerium oleander. Cette dernière espèce végétale est moyennement tolérante et accumule le sodium dans les parties basses sans que sa croissance en souffre (Hajji, 1979). Pour $P$. adenanthus, les teneurs en $\mathrm{Na}^{+}$des feuilles sont toujours plus élevées que celles des tiges et des racines. Ainsi $P$. adenanthus réagit comme la plupart des halophytes qui transfèrent aisément le sodium des racines vers les feuilles (Flowers et al., 1977 ; Brun, 1988 ; Suarez et al., 1998).

L'accumulation de $\mathrm{K}^{+}$est plus importante dans les feuilles que les tiges et les racines. L'apport de $\mathrm{NaCl}$ dans le milieu baisse de façon notable l'alimentation en $\mathrm{K}^{+}$des différents organes végétaux. Ces résultats rappellent ceux obtenus chez Triticum aestivum où la déficience en $\mathrm{K}^{+}$ est liée à la fois à l'ion ammonium et à la salinité du milieu (Al-Mutawa et El-Katony, 2001). La baisse de croissance des organes végétaux semble être liée en partie à la déficience des tissus foliaires en $\mathrm{K}^{+}$comme c'est le cas chez Nerium oleander (Hajji, 1979). Cette baisse de croissance peut aussi s'expliquer par un déficit hydrique dans les parties aériennes, lié au blocage du sodium dans les parties basses; le sodium n'étant pas transféré suffisamment jusqu'aux feuilles pour l'ajustement osmotique.

Le rapport $\mathrm{Na}^{+} /\left(\mathrm{Na}^{+}+\mathrm{K}^{+}\right)$montre une forte rétention du sodium dans les racines et les tiges des espèces étudiées par rapport aux feuilles, sauf chez $P$. adenanthus où on observe un phénomène inverse. Ces observations confirment les conclusions obtenues dans le cas de $\mathrm{Na}^{+}$.

\section{ETAT HYDRIQUE}

La consommation hydrique moyenne en milieu non salé des plantules de G. max, M. poggei, $P$. vulgaris et $V$. unguiculata est supérieure à celle de $P$. adenanthus. Chez M. poggei, $P$. adenanthus et $V$. unguiculata, le taux d'inhibition du sel reste relativement modéré. Ces résultats confirment ceux énoncés par Lötschert (1970) et Brun (1987). Selon ces auteurs, l'absorption de l'eau est plus importante chez les glycophytes sensibles que chez les halophytes. Par ailleurs, l'augmentation de la salinité diminue la perméabilité des racines comme c'est le cas chez les glycophytes et quelques halophytes (Kaplan et Gale, 1972).

\section{CONCLUSION}

Le comportement des espèces végétales étudiées dans les différents milieux expérimentalement enrichis en sel permet de distinguer les «glycophytes sensibles» (Glycine max et Phaseolus vulgaris) des espèces moyennement tolérantes (Mucuna poggei et Vigna unguiculata) ou tolérante (Phaseolus adenanthus). Le bon comportement de $M$. poggei, $V$. unguiculata et $P$. adenanthus permet d'envisager leur utilisation comme espèces pionnières sur des terres plus ou moins salées. Ces espèces faciliteraient l'implantation ultérieure d'autres cultures moyennement résistantes au sel.

Nos résultats portent sur le stade végétatif de 5 espèces végétales. Ils devront être complétées par les observations au stade floraison, afin de rendre compte de l'influence du stress salin sur la totalité de leur cycle de culture. 


\section{REFERENCES}

AL-MUTAWA (M. M.) and (T. M.) EL-KATONY. 2001. Salt tolerance of two wheat genotypes in response to the form of nitrogen. Agronomie $21: 259-266$.

BERGER (A.). 1978. L'alimentation en eau en milieu salé. Soc. Bot. Fr., Actual bot. 125 : 159-176.

BRUN (A.). 1981. Mise au point bibliographique concernant l'étude des effets de la salinité sur les végétaux. Ann. Fac. Sci. 28 : 59-84.

BRUN (A.). 1987. Importance relative des différents effets de $\mathrm{NaCl}$ sur la croissance de deux légumineuses à graines du Cameroun : Phaseolus vulgaris L. (variété «Penkou») et Canavalia obtusifolia DC. Ann. Fac. Sc. Biochim. $1:$ 37-48.

BRUN (A.). 1988. Effets de $\mathrm{NaCl}$ sur une halophyte tropicale (Canavalia obtusifolia DC.) : croissance, distribution de $\mathrm{Na}^{+}$et $\mathrm{K}^{+}$, état hydrique. Oecol. Plant. 9 : 173-186.

DUKE (A.). 1983. Handbook of legumes of world economic importance. Plenum Press, N.Y. and London, $345 \mathrm{p}$

EL-IKLIL (Y.), (M.) KARROU and (M.) BENICHOU. 2000. Salt stress effect on epinasty in relation to ethylene production and water relations in tomato. Agronomie 20 : 399-406.

FLOWERS (T. J.), (P. F.) TROKE and (A. R.) YEO. 1977. The mechanism of salt tolerance in halophytes. Ann. Rev. Plant Physiol. 28 : 89-121.

GREENWAY (H.) and (R.) MUNNS. 1980. The mechanism of salt tolerance in nonhalophytes. Ann. Rev. Plant Physiol. 31: 149-190.

HAJJI (M.). 1979. Effets du sel sur la croissance et l'alimentation minérale du Laurier-rose. Physiol. Veg. 17 : 517-524.

JACOBY (B.). 1964. Fonctions of bean roots and stems in sodium retention. Plant Physiol. $39: 445-449$.

KHADRI (M.), (L.) PLIEGO, (M.) SOUSSI, (C.) LLUCH and (A.) OCAÑA. 2001. Ammonium assimilation and ureide metabolism in common bean (Phaseolus vulgaris) nodules under salt stress. Agronomie 21 : 635-643.

KAPLAN (A.) and (J.) GALE. 1972. Effets of sodium chloride salinity on the water balance of Atriplex halimus. Aust. Journ. Biol. Sci. 25 : 895-903.

LESSANi (H.) and (H.) MARSCHNER. 1978. Relation between salt tolerance and long-distance transport of sodium and chloride in various crop species. Aust. J. Plant Physiol. $5: 27-37$.

LEVITT (J.). 1980. Responses of plants to environmental stresses. Vol. II : water, radiation, salt and other stresses. United Kingdom Edition Academic Press, London, 395-434.

LÖTSCHERT (W.). 1970. Keimung, transpiration, wasserund ionenaufnahme bei glycophyten und halophyten. Oecol. Plant. 5 : 287-300.

OKUSANYA (O. T.). 1980. The effect of salinity and nutrient level on the growth of Lavatera arborea. Oïkos 35 : 49-54.

PASQUET (R.). 1984. Les Légumineuses alimentaires du Cameroun. Mémoire ORSTOM, Yaoundé, $78 \mathrm{p}$

RAJESH (A.), (R.) ARUMUGAM and (V.) VENKATESALU. 1998. Growth and photosynthetic characteristic of Ceriops roxburghiana under $\mathrm{NaCl}$ stress. Photosynthetica 35 : 285-287.

SAADALLAHA (K.), (J. J.) DREVONB, (M.) HAJJIC and (C.) ABDELLYA. 2001a. Genotypic variability for tolerance to salinity of N2-fixing common bean (Phaseolus vulgaris). Agronomie $21: 675-682$.

SAADALLAHA (K.), (J. J.) DREVONB and (C.) ABDELLYA. 2001b. Nodulation et croissance nodulaire chez le haricot (Phaseolus vulgaris) sous contrainte saline. Agronomie 21 : 627-634.

SAINTILAN (N.). 1998. Relationships between height and girth of mangrove and soil-water conditions in the Mary and Hawkersbury River estuaries, eastern Australia. Aust. J. Ecol., 322-328.

SAVOURÉ (J. C.). 1980. Manipulations pratiques en physiologie végétale. Masson, Paris, $258 \mathrm{p}$.

SCHEIDECKER (D.). 1978. Réactions au sel d'un glycophyte : l'exemple du haricot. Soc. Bot. Fr., Actual. Bot. 125 : 137-147.

SHAPMAN (V. J.). 1976. Mangrove vegetation. J. Cramer, Vaduz, $447 \mathrm{p}$.

SLAMA (F.). 1987. Recherches sur les causes de l'exclusion du sodium des feuilles des plantes sensibles à $\mathrm{NaCl}$. Agron. Trop. 7 : 517522.

SLAMA (F.) and (E.) BOUAZi. 1978. Absorption et distribution interne du sodium chez le soja cultivé en milieu salé. Agrochemica 22 : 128-133. 
SOBRADO (M. A.). 1999a. Leaf photosynthesis of the mangrove Avicennia germinans as affected by $\mathrm{NaCl}$. Photosynthetica, 36 : 547555.

SOBRADO (M. A.). 1999b. Drought effects on photosynthesis of the mangrove, Avicennia germinans, under contrasting salinities. Trees $13: 125-130$.

SOBRADO (M. A.) and (M. C.) BALL. 1999. Light use in relation to carbon gain in the mangrove, Avicennia marina, under hypersaline conditions. Aust. J. Plant Physiol. 26 : 245251.

SUÁREZ (N.), (M. A.) SOBRADO and (E.) MEDINA. 1998. Salinity effects on the leaf water relations components and ion accumulation patterns in Avicennia germinans (L.) seedlings. Oecologia 114 : 299-309.

TATTINI (M.), (R.) GUCCI, (M. A.) CORA-DESCHI, (C.) PONZIO, and (J. D.) EVERARD. 1995.
Growth, gas exchange and ion content in Olea europaea plants during salinity stress and subsequent relief. Physiologia Plantarum 95 : 203-210.

TSOATA (I.) et (V. D.) TAFFOUO. 1997. Absorption du sodium par les parties aériennes de quatre espèces de Légumineuses. Agron. Afr. $9: 37-43$

VENKATARAMAN (S.) and (G. S.) VENKATARAMAN. 1995. Response of Anabaena to salt stress. 3. Effect of exogenous nitrogen. Nat. Acad. Sci. Letters India 18 : 165-166.

WACQUANT (J. P.). 1974. Recherche sur les propriétés d'absorption cationique des racines. Rôle physiologique et importance écologique. Thèse de doctorat, Montpellier, $155 \mathrm{p}$. 